\section{A new 3D hybrid model for epithelial morphogenesis}

Fithelial tissues line most of the insight into epithelial morphogents

Exithelial tissues line most of the $\quad$ insight into epithelial morphogenesis Thedy as well as our organs and blodvels. The membrans tisues cells that form barriers, thereby providng safety shields. Our skin, for example, is made up of millions of epithelial cells that are packed tightly together and provide a barrier between the inside and outside of our body. When epithelial tissues are wounded and the cells are destroyed or damaged, the existing cells and tissue drive the process of wound healing. Mechanical forces are also important for changing cell and tissue shape. The biological mechanism of wound closue enables the integrity and functionality of tissues to be preserved.

Epithelial morphogenesis encompasses a wide range of complex and diverse or intercalation, of epithelial tissue contributes to organ formation and body shape. Cell-cell interaction forces drive shape changes, and cell-based. biomechanics models can provide

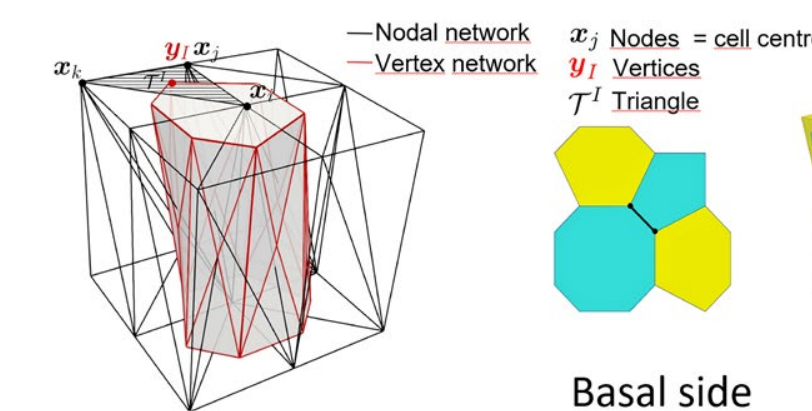

Basal side
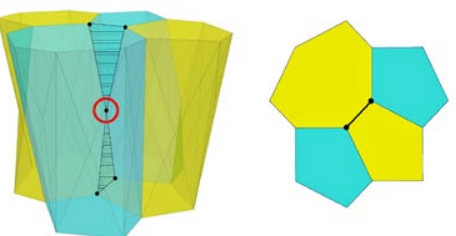

lateral side Apical side The 3D model approximates cell shape with a vertex network (in red)
and a nodal network (in black) representing cell-centre connections.
Professor at the Laboratory of Numerical Analysis (LaCâN), Universitat Politècnica dimensional mechanisms of epithelial morphogenesis that are involved in wound healing. In collaboration with Dr Filippos loannou, Dr Rob Tetley and Dr Yanlan Mao, all from University College London, and Malik A. Dawi, from the Universitat Politecnica de Catalunya, Dr Muñoz has developed a new 3D model for epithelial morphogenesis.

MODELLING

MORPHOGENETIC EVENTS Various modelling approaches have been developed to describe the cellto-cell interactions involved in epithelial morphogenesis. Processes such as the be described as planar of corms can and studied using two-dimensionat tools and vertex models. These vertex models approximate the cell shape with a two-dimensional polygon representation of the cellular interfaces where vertices mark the common points with neighbouring cells. The vertices move in response to forces during from the balance of forces produced by cell-cell adhesion, and pressure within each cell. Dr Munoz explains how in order to understand the forces in these processes, we need models that are capable of reproducing both junctional mechanics and cell deformations that basal (bottom) sides of the monolayer, growth, interfacial tension resulting

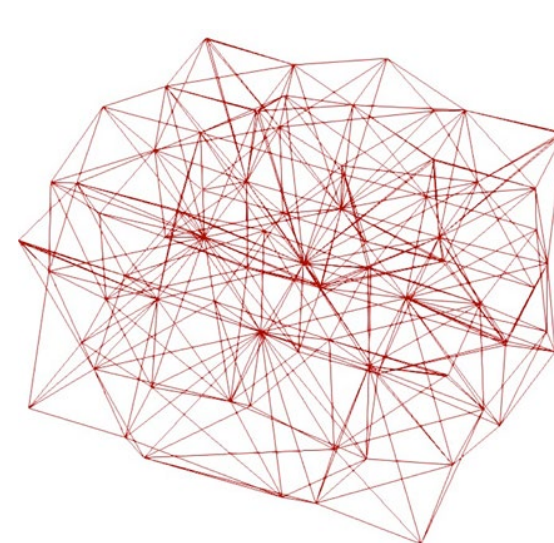

3D model of cell aggregates.

to represent a single, closely packed layer of cells.

THREE-DIMENSIONAL MECHANISMS DrMuñoz observes that while the twodimensional analysis of wound healing known about the dici, much less is mechanisms that are involved. He explains how many other epithelial shape changes, such as bending tissue folding cell extrusion and delamination, can be described using out-of-plane mechanics and three-dimensional effects. Tissue closure involves a combination of contractile forces on the top, or apical region of the wound. This has motivated Dr Muñoz and his collaborators to develop a threedimensional model that enables the simulation (the cells researchers can study their effects on wound closure.

\section{D HYBRID VERTEX MODEL}

Dr Muñoz had previously been involved in the development of a two-dimensiona model, which the researchers have extended to three dimensions. This new 3D model is a hybrid between a cell-centred model, where each cell is depicted as a sphere, or node, and each cell-cell interaction is modelled with a line or bar element, connecting two nodes, and a pure vertex model, where the cell cortex is represented as a polygon, with vertices and edges shared with adjacent cells. The new cell bounder but tit to
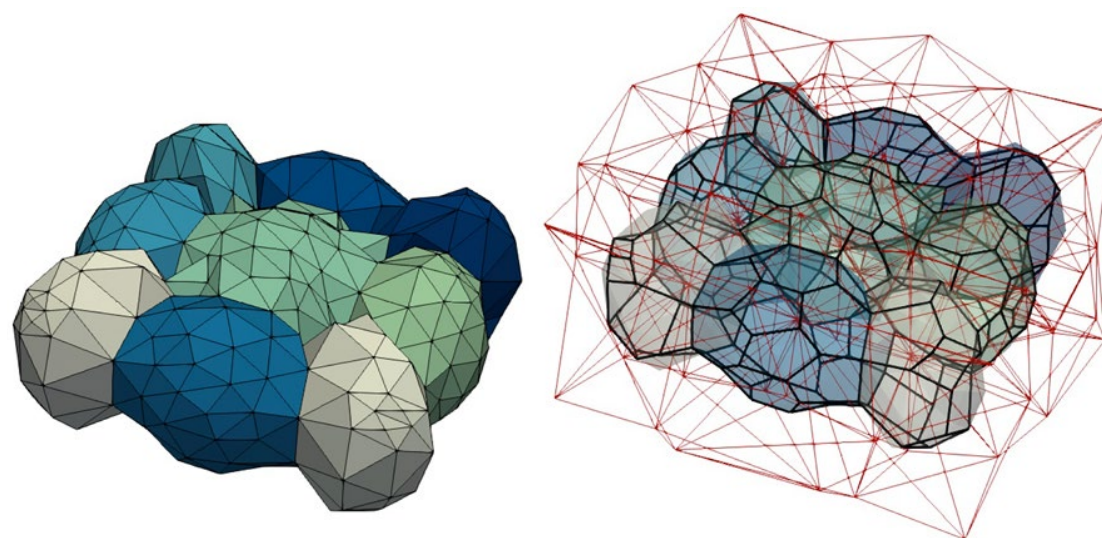

centres as degrees of freedom in order to describe how the whole cell changes shape, i.e. the whole cell kinematics.

Monolayer geometry is used to define the cells. The positions of the cell centres are taken from two-dimension experimental images and provide basal cell centres in the model. The initial locations of the cell centres and the shape of the cell areas at both the apical and basal surfaces are equal.

WOUNDING AND CONTRACTILITY EXPERIMENT The researchers carried out an experiment to evaluate the model's simulated epithelial morphogenesis. Tormation of a scar. Embryonic and lanval tissues, however, have the ability to complete the healing process

in a seamless manner. Larval tissues

of Drosophila (a small fruit fly) were chosen for the investigation. The Drosophila larval wing imaginal disc is highly columnar cells. of wound healing has been well highly columnarcells. tudied, much less is known about the be arranged in layers, three-dimensional mechanisms that attached to the are involved.

In subsequent time-steps, however they can independenty change their dimensional shapes with polygons as faces) that can have different shapes and number of sides at their apical and basal surfaces. The model computes the positions of the vertices at the apical. and basal surfaces resorting to nodal interpolation. Mechanical equilibrium is achieved by minimising the total energy with respect to the positions of the nodes and the vertices around the edge of the wound. The cells' viscoelastic behaviour is modelled using a rheological model that obeys a rheological law based on rest-length changes. The num found by solving the resulting system Raphson iterative process. experimental system

behaviours in three dimensions.

The wing disc epithelia was ounded using a pulsed

TSa laser, and images

a

Dr Muñoz modelled larval tissue of the fruit fly. experiments in ideal 

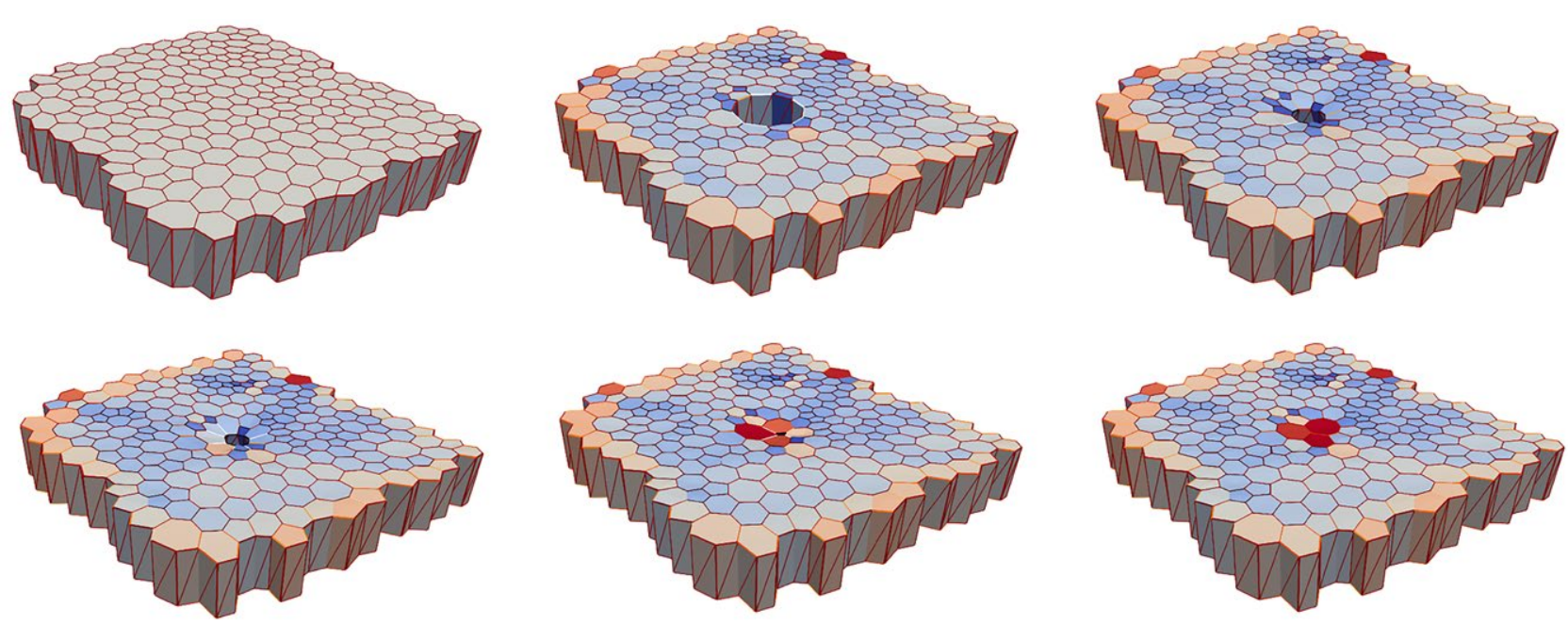

of the wound healing response were recorded every 3 minutes using 3D me-lapse confocal microscopy. A similar the softening and removal of degraded cells and the progressive wound closure simulated with a contraction at the wound front and lateral faces, and a subsequent intercalation (changing places) of the cells at the wound edge.

WOUND HEALING SIMULATIONS Experimental

In addition to tissue

actomyosin purse-string - a protein complex that is able to contract to draw edges of the wound inward.

The researchers tested a variety of simulated patches ranging in size from 80 for each of the cases that had been measured experimentally. They measured and compared the time evolution of the

ANALYSIS

simulations with experimental results revealed that in addifion to tissue tension and driven by the rate of intercalation, issue fluidity. Pr Muñoz describes how the inclusion of lateral contractility, together with the simulation of diffe intercalation at both the apical and measurements were taken of wounds in five separate wing discs and averaged. The evolution of the wing disc apical wound area was quantified by manually tracing the 72 perinery of the wound for the first 72 min after wounding. The wound penphe while pand healing progess due to the formation of an apical

Drosophila imaginal disc wound healing

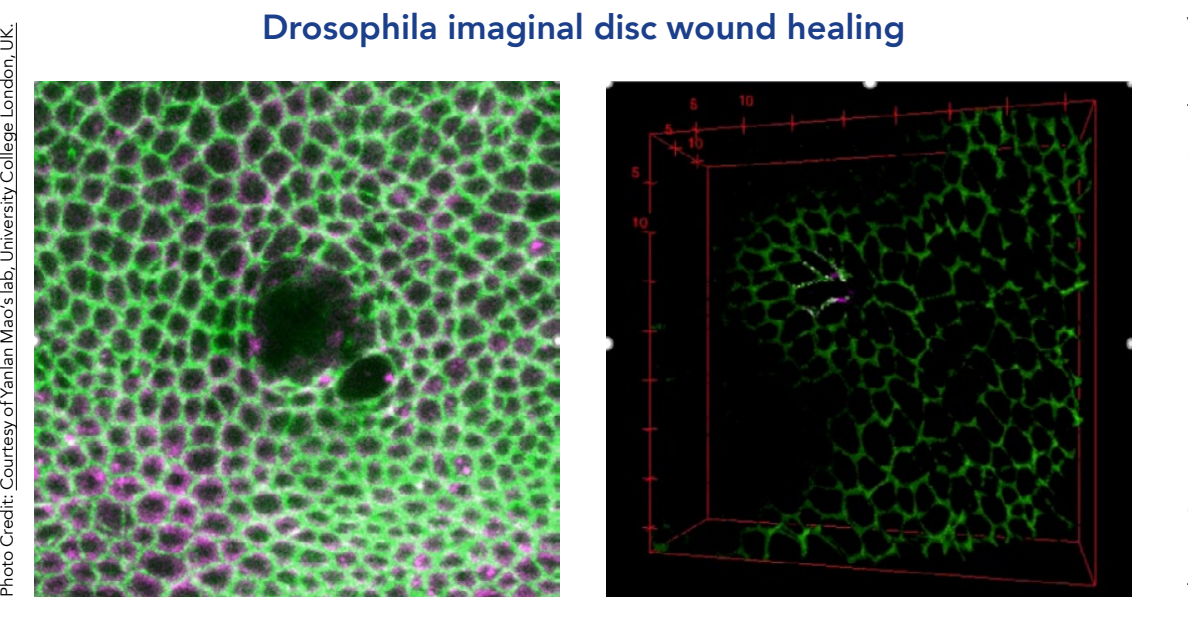

Cell boundaries after wounding (left) and three-dimensional view of the apical surface at the top
of monolayer (right), showing concentration of myosin in violet.

\section{by the rate of intercalation.}

relative projected area and the relative height of the wound edge in both the experimental images and the simulation The evolution of the inting took place. also andyed in terms of the number of to test the effects of the wound edge closure. The numbions on wound indicated that applying apical pursestring tension together with volume preservation induced a reduction of the relative height at the wound edge, due to the expansion of the tissue. Dlume preservation is a fundamental sideration as significant changes to the cell volume compromises both its structural integrity and the constancy of the death of the cell.

Dr Muñoz explains how the simulation 作 the three-dimensional mechanisms of wound closure with a vertex model, supported by experimental observations, enabled the researchers to the the simulation results reveal. Analysing full closure is to be achieved. The researchers intracellular environment. This can result necessity of cell volume preservation if

\section{Behind the Research}

\section{Dr Jose Muñoz}

E: j.munoz@upc.edu T: +34934137253 W: https://www.lacan.upc.edu/jose.munoz/

\section{Detail}

Jose Muñoz

Escola d'Enginyeria Barcelona Est (EEBE)

Universitat Poltiècnica de Catalunya

Av. Eduard Maristany 16

Bio

Jose J Muñoz studied Mechanical Engineering at

Con

PhD in computational mechanics at Imperial College

London. Since then, he has investigated and modelled

mechanical problems with emphasis in biological

applications: embryogenesis and tissue mechanics.

\section{Funding}

Muñoz and Malik Dawi have been financially supported by the Spanish Ministry of Science, Innovation and Universities (MICINN) with grant Generalitat de Catalunya with grant 2017 SGR 1278. Rob Tetley was funded by a Medical Research Council Skills Development Fellowship (MR/NO14529/1). Yanlan Mao was funded by a Medical Research Council Fellowship MR/L009056/1, a Lister Institute Research Prize, and EMBoroung livestigator Programme. Filizos loannou was a lso supported by Medical Research Coun funding to Biology (LMCB) Univasity Unt at University College London, award code MC_U12266B.

\section{Collaborators}

nou (UCL, LMCB)

- Malik A. Dawi (UPC)

- Robert J. Tetley (UCL, LMCB)

- Yanlan Mao (UCL, LMCB, Nanjing University, China)

\section{Research Objectives}

Dr Muñoz analyses the three-dimensional mechanism involved in wound healing.

\section{References}

loannou, F., Dawi, M.A., Tetley, R.J., Mao, Y., Munoz, J.J. (2020). Development of a new 3D hybrid model for

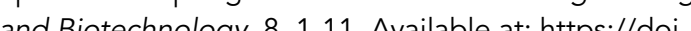
org/10.3389/fbioe.2020.00405

\section{Personal Response}

What inspired you to extend your original 2D model to this new 3D hybrid model?

II Discussions with scientists and observation of 3D images revealed that wound closure is far from a flat
vertical profile advancing at the wound front and with constant height. This new view posed new modelling challenges, but also opened a new hypothesis for effective tissue along cell height is progressively completed.
the 